\title{
Disease Activity of Juvenile Idiopathic Arthritis during and after Pregnancy: A Prospective Multicenter Study
}

\author{
Kristin Ursin, Stian Lydersen, Johan F. Skomsvoll, and Marianne Wallenius
}

\begin{abstract}
Objective. To study disease activity in women with juvenile idiopathic arthritis (JIA) during and after pregnancy. There is little previous knowledge about this topic.

Methods. Our study included 135 pregnancies in 114 women with JIA. Disease activity was assessed at 7 timepoints before, throughout, and after pregnancy with the Disease Activity Score-28-C-reactive protein 3 (DAS28-CRP3). Scores assessed at each visit were analyzed in a linear mixed model. The same statistical method was used to study self-reported physical function, pain, and mental health. Results. Almost $80 \%$ of the women were in remission or had low disease activity during and after pregnancy. Although disease activity was stable throughout the study period, we found that DAS 28 6 weeks postpartum increased significantly compared to the first trimester ( 2.78 vs $2.51, \mathrm{p}=0.005)$ and third trimester $(2.78$ vs $2.56, \mathrm{p}=0.011)$, respectively. DAS28 decreased significantly between 6 weeks and 12 months postpartum $(2.78$ vs $2.54, p=0.014)$. Self-reported mental health was significantly better 6 weeks postpartum than before pregnancy (Medical Outcomes Study Short Form-36 Mental Health subscale 80.7 vs $76.5, p=0.039$ ). Self-reported pain was stable. Physical function was significantly worse in the third trimester of pregnancy than postpartum (Modified Health Assessment Questionnaire 0.57 vs $0.39, \mathrm{p}<0.001$ ).

Conclusion. In women with JIA, disease activity was highest 6 weeks postpartum, but altogether low and stable in the period from planning pregnancy to 1 year after delivery. (First Release December 1 2017; J Rheumatol 2018;45:257-65; doi:10.3899/jrheum.161410)
\end{abstract}

Key Indexing Terms: JUVENILE IDIOPATHIC ARTHRITIS

Juvenile idiopathic arthritis (JIA) is the most common chronic inflammatory rheumatic disease in childhood, and it affects twice as many girls as boys. A study from 2003 reported an incidence of JIA of 15 per 100,000 per year in Nordic countries ${ }^{1}$. More than $40 \%$ of women with JIA have

From the National Advisory Unit on Pregnancy and Rheumatic Diseases, Department of Rheumatology, Trondheim University Hospital; Department of Neuromedicine and Movement Science, Faculty of Medicine, Norwegian University of Science and Technology (NTNU); Regional Center for Child and Youth Mental Health and Child Welfare, Faculty of Medicine, NTNU, Trondheim, Norway.

Supported by the Department of Rheumatology, Trondheim University Hospital, and the Research Fund of the Norwegian Organization for People with Rheumatic Diseases.

K. Ursin, MD, National Advisory Unit on Pregnancy and Rheumatic Diseases, Department of Rheumatology, Trondheim University Hospital, and Department of Neuromedicine and Movement Science, Faculty of Medicine, NTNU; S. Lydersen, PhD, Regional Center for Child and Youth Mental Health and Child Welfare, Faculty of Medicine, NTNU;

J.F. Skomsvoll, MD, PhD, National Advisory Unit on Pregnancy and Rheumatic Diseases, Department of Rheumatology, Trondheim University Hospital; M. Wallenius, MD, PhD, National Advisory Unit on Pregnancy and Rheumatic Diseases, Department of Rheumatology, Trondheim University Hospital, and Department of Neuromedicine and Movement Science, Faculty of Medicine, NTNU.

Address correspondence to Dr. K. Ursin, National Advisory Unit on Pregnancy and Rheumatic Diseases, Department of Rheumatology, Trondheim University Hospital, Postbox 3250 Sluppen, NO-7006 Trondheim, Norway.E-mail: kristin.ursin@ntnu.no

Accepted for publication September 8, 2017.

\section{DISEASE ACTIVITY PREGNANCY}

active disease or are taking medication ${ }^{2,3}$ while of childbearing age.

A well-designed study from 2008 confirmed that women with rheumatoid arthritis (RA) achieve remission in pregnancy, but less frequently than previously described ${ }^{4}$. The only studies on disease activity in pregnancy in women with JIA date to the 1990s, before biological disease-modifying antirheumatic drugs (bDMARD) were available ${ }^{5,6}$. Both studies were based on questionnaires in which women were asked in retrospect about disease activity during and after pregnancy. The majority reported lower disease activity in pregnancy, while about $50 \%$ reported higher disease activity postpartum.

The main aim of our study was to prospectively study disease activity in women with JIA during pregnancy and until 12 months postpartum using a validated disease activity measure. In addition, we wanted to explore the women's self-reported physical function, pain, and mental health throughout the study period.

\section{MATERIALS AND METHODS}

RevNatus register. RevNatus is a Norwegian nationwide register designed for the followup of women with inflammatory rheumatic diseases from the planning of pregnancy until 1 year postpartum. The register was established at the Norwegian National Advisory Unit on Pregnancy and Rheumatic Diseases in 2006.

Patient population. Our study comprises women with JIA included in RevNatus in the period from January 1, 2006, to November 17, 2015. All 
women were diagnosed by a rheumatology specialist according to the International League of Associations for Rheumatology criteria for JIA ${ }^{7}$. RevNatus does not contain data on JIA subtypes.

All women with JIA who contributed data from at least 1 timepoint in the period from the first trimester to 1 year after delivery were included in the study. We excluded women who did not conceive within 1 year after inclusion and women who had a miscarriage.

Data collection and description of outcome variables. Women included in RevNatus ideally have 7 visits at their local rheumatology unit: before conception (visit 0 ), in each trimester (visits 1-3), and 3 times postpartum (at 6 weeks, 6 months, and 12 months after delivery; visits 4-6). The objective is that all women of childbearing age diagnosed with an inflammatory rheumatic disease are told to contact their rheumatology unit when planning pregnancy to be included in RevNatus. However, only a minority of the women with JIA were in fact enrolled preconception. Thus, not all women attended all visits. Enrollment was carried out by rheumatologists and nurses at each participating center.

At each visit, local rheumatologists evaluated disease activity using Disease Activity Score-28-C-reactive protein 3 (DAS28-CRP3). The score is composed of a 28 -joint count for swelling and tenderness combined with the CRP level ${ }^{8}$. DAS28 is a commonly used disease activity measure in patients with arthritis, and DAS28-CRP3 is validated for use during pregnancy in women with $\mathrm{RA}^{9}$. CRP levels were measured by local methods. All values $<5 \mathrm{mg} / \mathrm{l}$ (the lower detection limit) were defined as $4 \mathrm{mg} / \mathrm{l}$ in the calculation.

The European League Against Rheumatism (EULAR) defines 4 disease categories according to the DAS28 score: remission (DAS28 $\leq 2.6$ ), low disease activity $(2.6<$ DAS2 $2 \leq 3.2)$, moderate disease activity $(3.2<$ DAS28 $\leq 5.1$ ), and high disease activity (DAS28 $>5.1)^{10}$

Self-reported scores of the Medical Outcomes Study Short Form-36 (SF-36) and the Modified Health Assessment Questionnaire (MHAQ) were also collected at each visit. The SF-36 consists of 36 questions in 8 health-related dimensions, resulting in 1 score in each dimension with a value $0-100(100=\text { best possible health })^{11}$. We studied 3 of the dimensions: physical functioning, bodily pain, and mental health. RevNatus uses SF-36 version 1.0. MHAQ is composed of 1 question from each of the original 8 HAQ categories, all describing the ability to perform a certain practical task on a 4-point Likert scale (from $0=$ "without difficulty" to $3=$ "unable to do") ${ }^{12}$.

Data on positivity for rheumatoid factor (RF IgM, RF IgG, and/or RF $\operatorname{IgA})$, anticyclic citrullinated peptide antibodies, and antinuclear antibodies were collected in the first trimester.

Information about medication was collected at each visit. We divided medication into oral steroids, synthetic DMARD (sDMARD), and bDMARD.

Data and statistical analysis. We calculated the proportion of women in each EULAR disease activity category at each timepoint. To find out how disease activity changed during and after pregnancy, we analyzed DAS28-CRP3 scores from 7 timepoints (visits 0-6) in a linear mixed model. The reference point was visit 4 (6 weeks postpartum), a well-defined nonpregnant timepoint with few missing DAS28 scores. We used a 3-level model where visits were nested within pregnancies, and pregnancies were nested within women. We did additional analyses, including the oral steroids (yes/no) and sulfasalazine (SSZ; yes/no) in the mixed model analysis, each at a time.

MHAQ scores and the chosen SF-36 dimensions at each timepoint were also analyzed in a linear mixed model. We considered a 2 -sided $\mathrm{p} \leq 0.05$ statistically significant. For statistical analysis, we used SPSS for Windows version 22.0 .

Ethics. The Regional Committee for Medical and Health Research Ethics approved this study in 2013 (REK 2013/649). All women included in RevNatus have given their informed written consent. They were treated according to established standards and were not subject to any experimental treatment. The study is in compliance with the Declaration of Helsinki.

\section{RESULTS}

Patient inclusion data. In the period between January 2006 and November 2015, RevNatus included 140 pregnancies in women with JIA. In addition, the registry included 27 women with JIA who did not conceive within 1 year after inclusion. Of the 140 pregnancies registered, 5 pregnancies ended in miscarriages. As shown in Figure 1, the study included a total of 135 pregnancies in 114 women with JIA. One woman had 3 pregnancies and 19 women had 2 pregnancies. One woman was pregnant with twins. One woman had no registered DAS28 scores and was excluded from the main analysis.

Not all women had data from all timepoints. The mean number of visits per pregnancy was 4.3. Only 47 women $(35 \%)$ had data from the preconception visit, and 5 women had no visits until after pregnancy.

Demographics, disease characteristics, and breastfeeding . The study population consisted of women with JIA with a median age of 29 years (range, 18-38 yrs) and median disease duration of 20 years (range, 4-36 yrs). Almost 50\% of pregnancies were in previously nulliparous women. Table 1 shows demographics and disease characteristics reported in the first trimester, and the proportion of women breastfeeding.

Evaluation of disease activity according to DAS28-CRP3. Figure 2 shows the percentage of women in each EULAR disease category at each timepoint. We found that almost $80 \%$ were in remission or had low disease activity from planning pregnancy to 1 year after delivery.

After analyzing DAS28-CRP3 values from 7 timepoints (visits 0-6) before, during, and after pregnancy in a linear mixed model, we also found that disease activity was low and relatively stable throughout the study period. However, there was a significant relationship between disease activity and timepoint in the followup period ( $\mathrm{p}<0.001)$. DAS28 6 weeks after delivery was significantly higher than in the first trimester $(2.78$ vs $2.51, \mathrm{p}=0.005)$, third trimester (2.78 vs $2.56, \mathrm{p}=0.011)$, and 1 year after delivery (2.78 vs 2.54 , $\mathrm{p}=0.014)$. We found a nonsignificant decrease in DAS28 between the preconception visit and first trimester (2.65 vs $2.51)$. Figure 3 shows changes in disease activity throughout the study period (panel A).

A change in DAS28 of $\geq 1.2$ is often regarded as clinically significant ${ }^{13}$. Of the women with data from both third trimester and 6 weeks postpartum, $22 \%$ had a postpartum increase in DAS28 $\geq 1.2$ and $4 \%$ had a postpartum decrease in DAS28 $\geq 1.2$, while the majority of the remaining women had a smaller increase in disease activity.

By including oral steroids as a variable in the mixed model analysis, we found that women using oral steroids had significantly higher DAS28 than women not using oral steroids (e.g., at 6 weeks postpartum, 3.09 vs 2.66 , p < 0.001). Women using SSZ also tended to have higher disease activity throughout the study period, but this was not a significant finding (results not shown). 


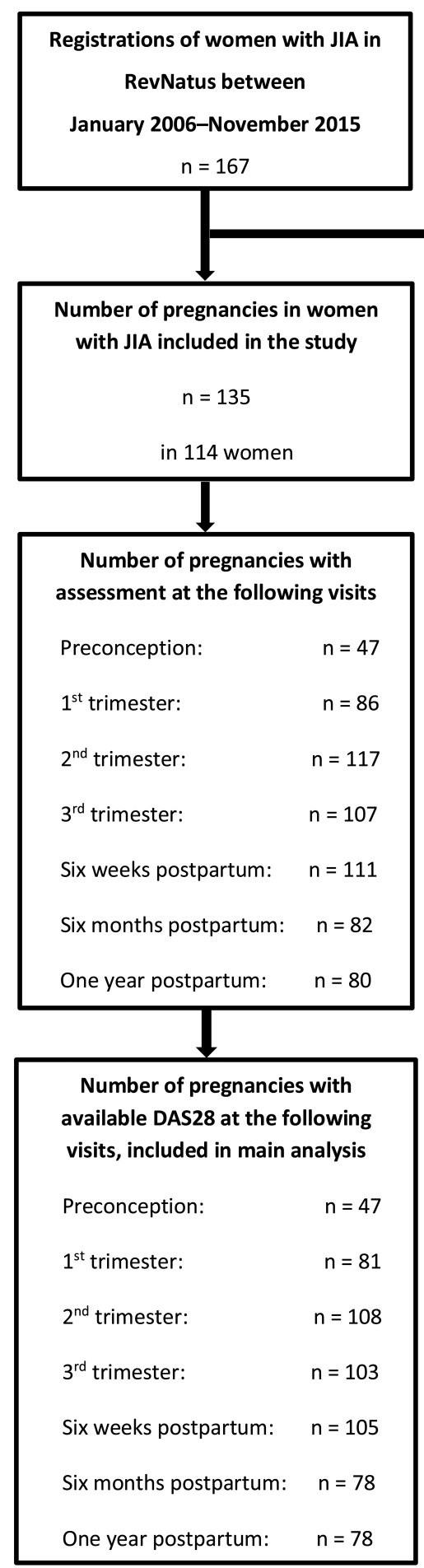

Not pregnant during follow up: $n=27(16 \%)$

Miscarriage:

$\mathrm{n}=5(3 \%)$

Number of pregnancies in women

with JIA included in the study

$n=135$

in 114 women

Figure 1. Flow chart showing inclusion data and data available for main analysis. JIA: juvenile idiopathic arthritis; DAS28: 28-joint Disease Activity Score

Evaluation of physical function and aspects of quality of life. MHAQ values were highest in the third trimester, corresponding to lowest functionality. After analyzing MHAQ values from all 7 timepoints in a linear mixed model, we found that functionality in the third trimester was signifi- cantly worse than at 6 weeks postpartum $(0.57$ vs 0.39 , $\mathrm{p}<0.001)$. In line with this result, the physical functioning score of SF-36 was significantly lower in the third trimester compared to 6 weeks postpartum $(60.8$ vs $72.8, \mathrm{p}<0.001)$.

Bodily pain scores were lowest in the third trimester of

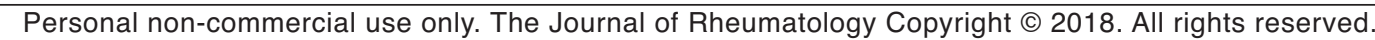


Table 1. Characteristics of study population at baseline, and proportion of breastfeeding. Values are mean \pm SD or $n(\%)$ unless otherwise specified.

\begin{tabular}{lc}
\hline Characteristics & Values \\
\hline Basic characteristics, $\mathrm{n}=86$ & \\
$\quad$ Age, yrs & $28.7 \pm 4.9$ \\
Disease duration, yrs & $20.0 \pm 6.9$ \\
Body mass index & $24.4 \pm 4.0$ \\
Smoking & $5(5.9)$ \\
Nulliparous* & $41(47.7)$ \\
Clinical characteristics, $\mathrm{n}=86$ & \\
RF present & $11(12.8)$ \\
ACPA present & $15(17.4)$ \\
ANA present & $11(12.8)$ \\
History of enthesitis & $1(1.2)$ \\
History of sacroiliitis & $2(2.3)$ \\
History of psoriatic skin disease & $1(1.2)$ \\
History of organ affection & $1(1.2)$ \\
Breastfeeding, $\mathrm{n} / \mathrm{N}(\%)$ & \\
6 wks after delivery & $86 / 111(77.5)$ \\
6 mos after delivery & $38 / 82(46.3)$ \\
1 yr after delivery & $10 / 80(12.5)$ \\
\hline
\end{tabular}

*Has never given birth to a live child. ACPA: anticyclic citrullinated peptide antibodies; ANA: antinuclear antibodies; RF: rheumatoid factor. pregnancy (bodily pain $=55.0$ ), corresponding to highest reported pain, but altogether reported pain was stable. The women reported significantly better mental health 6 weeks postpartum than before pregnancy (mental health $=80.7 \mathrm{vs}$ $76.5, \mathrm{p}=0.039$ ), and versus the first trimester (mental health 80.7 vs $76.4, p=0.006)$. Figure 3 shows changes in reported functionality, pain, and mental health throughout the study period (panels B, C, and D).

Medication use before, during, and after pregnancy. Table 2 shows the percentage of women using oral corticosteroids, sDMARD, and bDMARD in the year before pregnancy, during pregnancy, and in the year after delivery. The table also shows the percentage of women who discontinued 1 of these drugs before pregnancy.

The proportion of women using a DMARD (synthetic, biological, or both) decreased from $55 \%$ preconception to about $22 \%$ in pregnancy.

In the year before pregnancy, 24 women discontinued a sDMARD, of whom 20 had used methotrexate (MTX). Two of the women who discontinued MTX changed to hydroxychloroquine; the others stayed without an sDMARD. Of the women discontinuing an sDMARD before pregnancy, 21 women did so more than 3 months prior to conception.

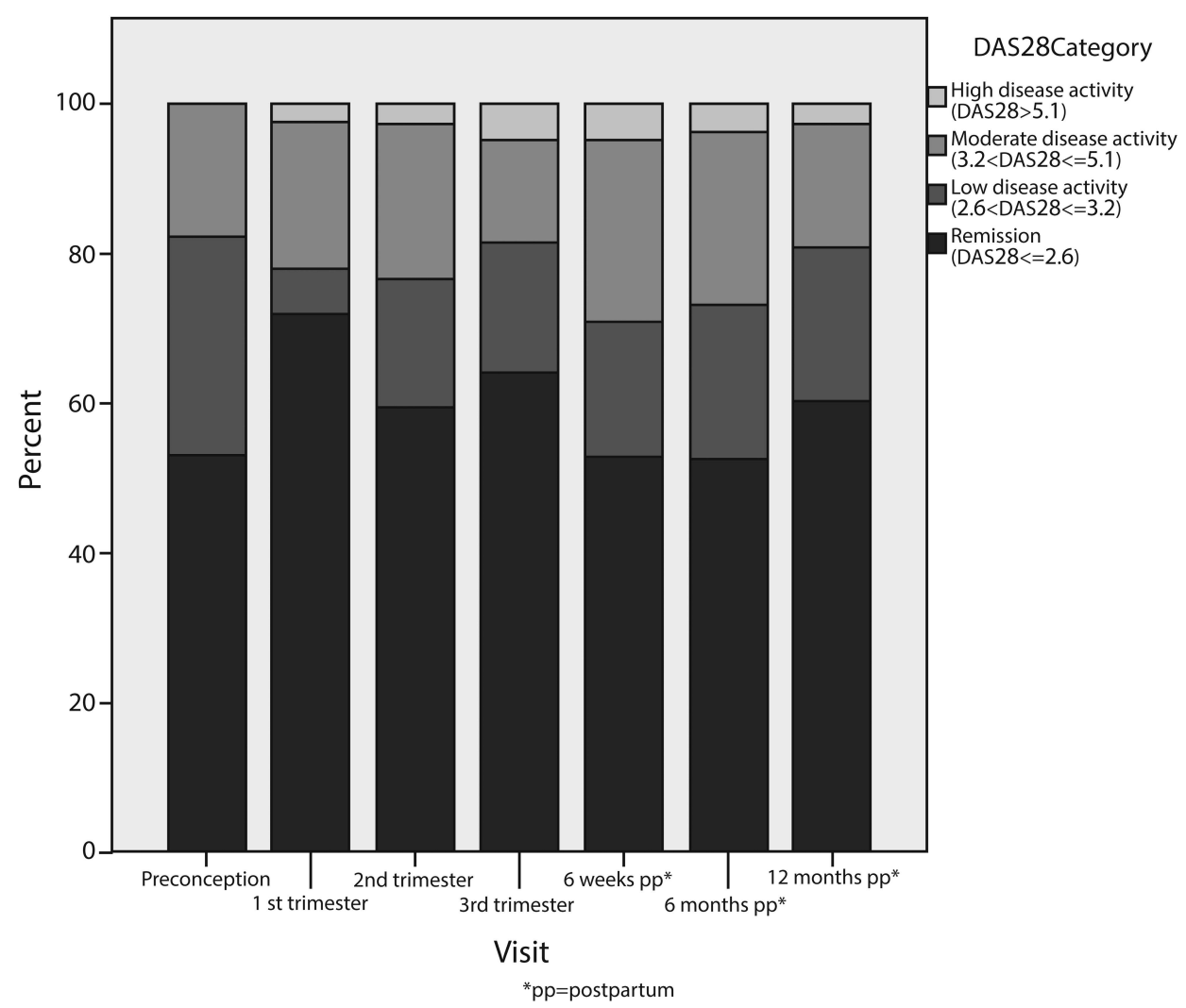

Figure 2. Percentage of women in each European League Against Rheumatism category before, during, and after pregnancy. DAS28: 28-joint Disease Activity Score. 

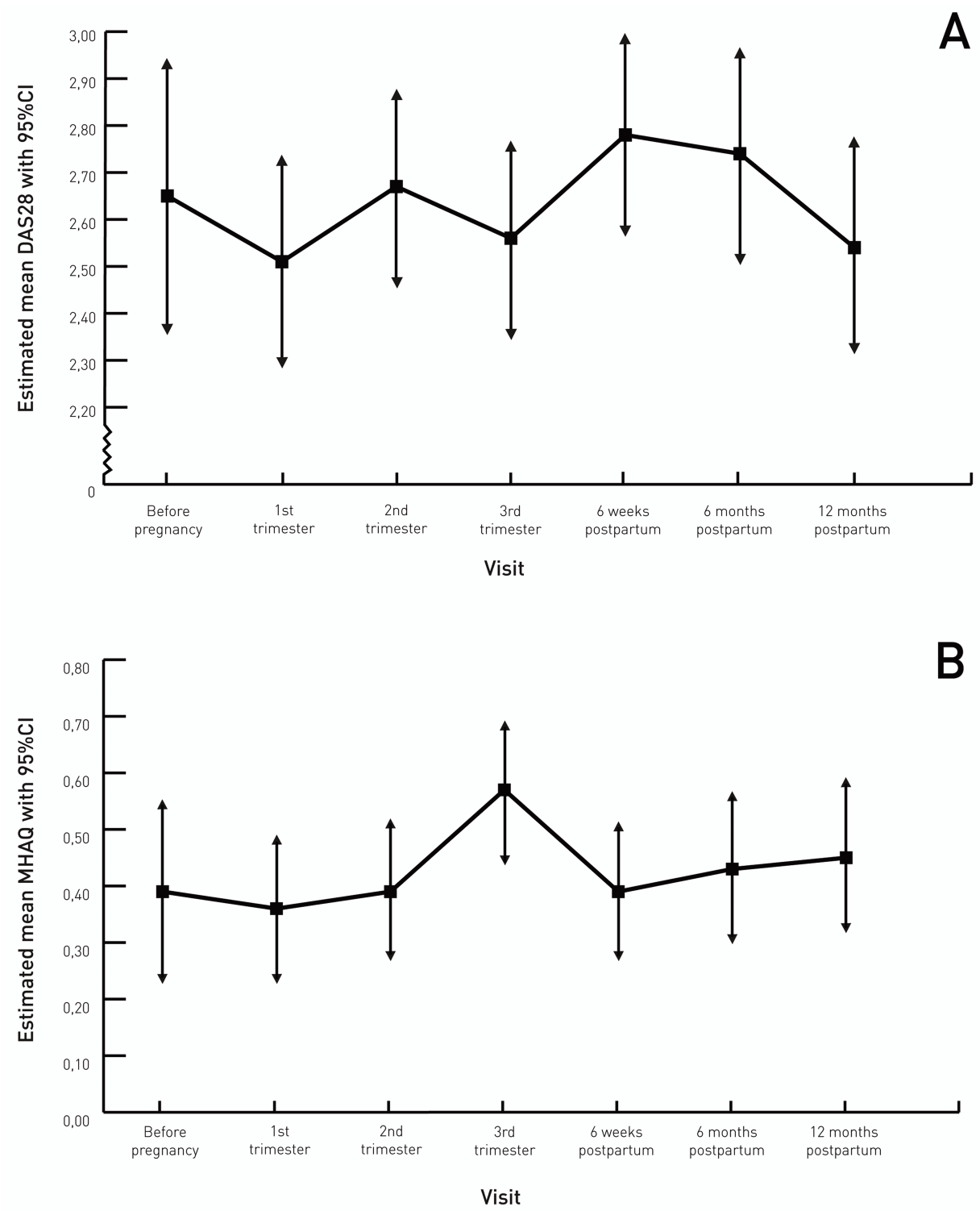

Figure 3. Estimated mean (A) DAS28-CRP3; (B) MHAQ; (C) SF-36 Bodily Pain scale; and (D) SF-36 Mental Health scale, with 95\% CI before, during, and after pregnancy. DAS28-CRP3: Disease Activity Score-28C-reactive protein 3; MHAQ: Modified Health Assessment Questionnaire; SF-36: Medical Outcomes Study Short Form-36.

Altogether, $18 \%$ of included women used sDMARD throughout pregnancy. Of these, $80 \%$ used SSZ, while the rest used hydroxychloroquine. One year postpartum, $40 \%$ used sDMARD, $>50 \%$ of whom used MTX (mostly the same women who used this drug prior to pregnancy). One-third of the women did not breastfeed 6 months postpartum, and this was related to the restart of MTX.

Altogether, 48 women discontinued bDMARD in the year prior to pregnancy. Of these women, 23 discontinued the drug $>3$ months prior to conception, 17 discontinued $<3$ months prior to conception, and 8 did so at confirmed pregnancy. Fewer than $5 \%$ of the women used bDMARD during pregnancy, all of whom used a tumor necrosis factor $\alpha$-inhibitor (3 out of 5 cases were women with uveitis). Most women using bDMARD in the year before and/or during pregnancy used the same bDMARD 6 and 12 months postpartum.

While there were 10 women (out of 47) taking oral steroids at the preconception visit, the numbers increased to 24 (out of 85) women in the first trimester and 30 (out of 81) women 6 months postpartum. Prednisolone doses before and during pregnancy were mostly kept $<10 \mathrm{mg}$ daily. Postpartum doses were $\leq 30 \mathrm{mg}$ daily.

\section{DISCUSSION}

In studying women with JIA from before pregnancy to 1 year postpartum, we found that almost $80 \%$ were in remission or had low disease activity throughout the study period. This is

$$
\text { Personal non-commercial use only. The Journal of Rheumatology Copyright (C) 2018. All rights reserved. }
$$




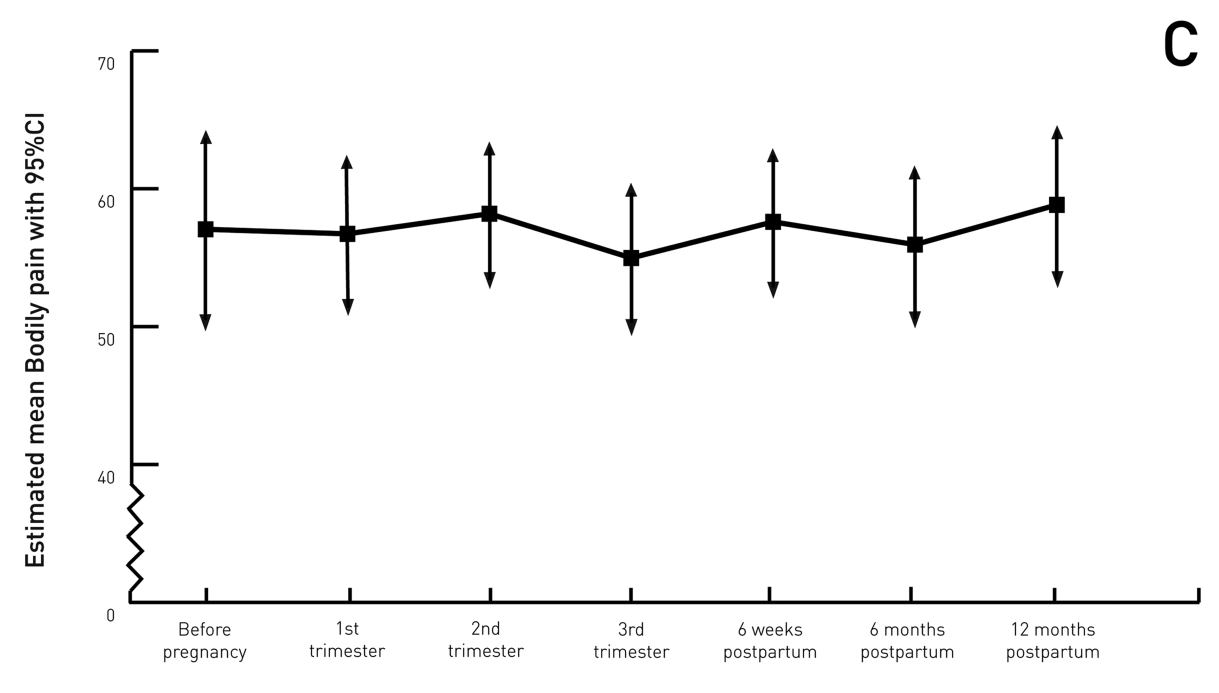

Visit

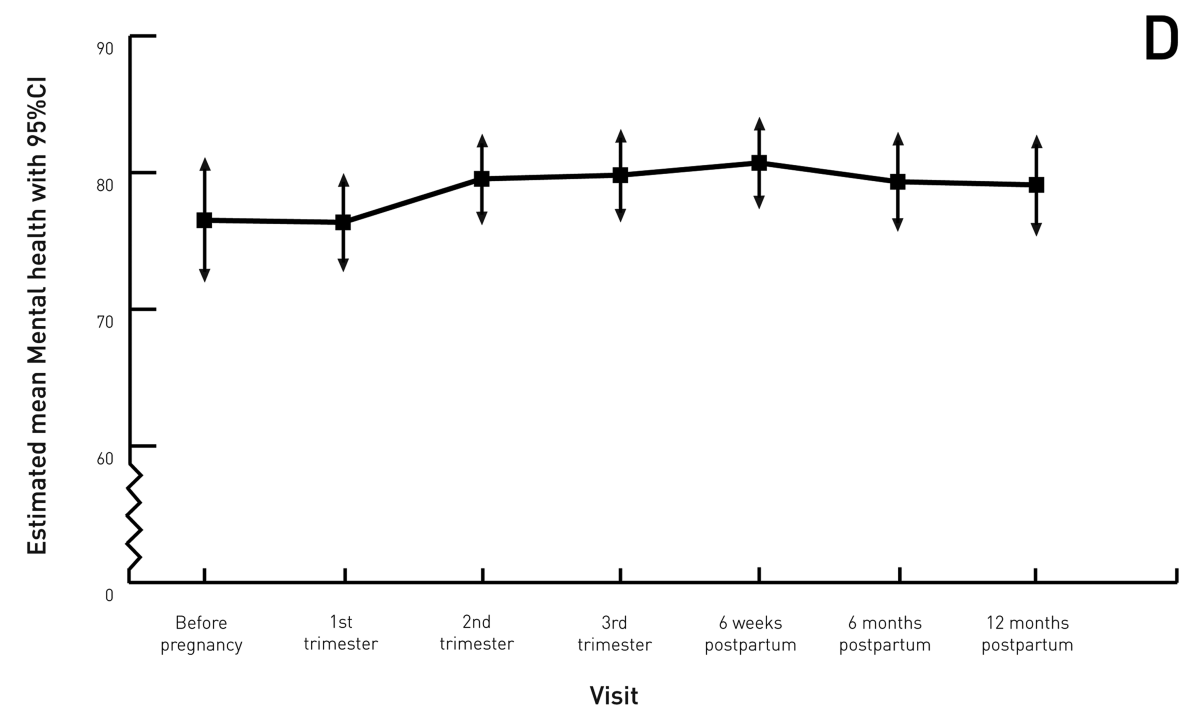

Figure 3. Continued

despite the proportion of women using DMARD (synthetic, biological, or both) decreasing from 55\% in the year prior to pregnancy to $22 \%$ during pregnancy. Altogether, disease activity was stable, but we found that disease activity 6 weeks after delivery was significantly higher than in the first trimester, third trimester, and 12 months postpartum.

To our knowledge, this is the first prospective study of disease activity during and after pregnancy in women with JIA, and the first to use a validated disease activity score instead of questionnaires and hospital records.

Two previous retrospective studies and 1 case series demonstrated that women in remission before pregnancy remained in remission during pregnancy, and that $>50 \%$ of the women with active disease at baseline experienced amelioration $^{5,6,14}$.

Our study population differed from those of previous studies: the majority had stable low disease activity, and many had planned pregnancies with treatment optimized at baseline and continued throughout pregnancy (22\% used DMARD). In the previous studies, more women had active disease at baseline and only 1 woman used DMARD ${ }^{5,6}$. In a population with a larger proportion of participants with active disease at baseline, it could be easier to detect relative amelioration. However, we cannot exclude a potential beneficial effect of pregnancy in our study population. With a large decrease in women taking DMARD, we would expect an 
Table 2. Medication use before, during, and after pregnancy. Values are n (\%).

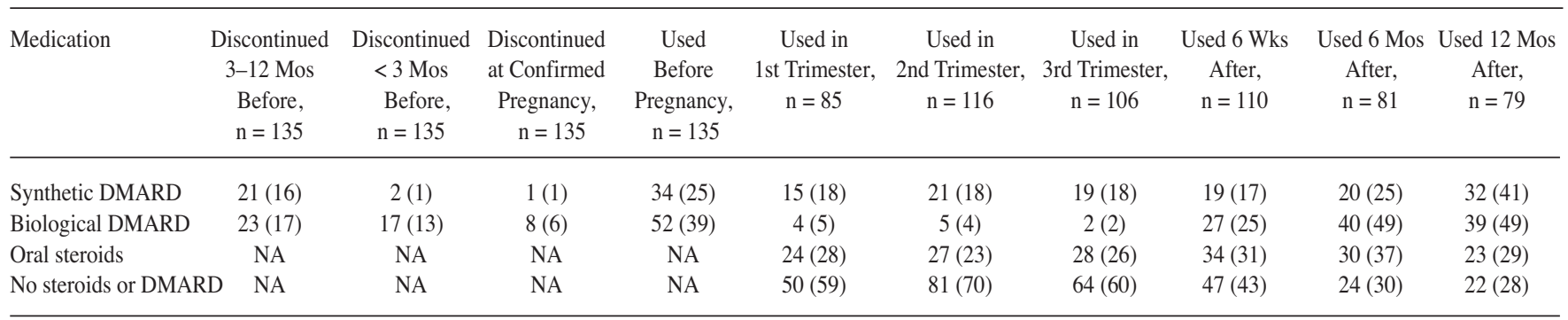

NA: not available; DMARD: disease-modifying antirheumatic drugs.

increase in disease activity. However, we found stable low disease activity, and even a small decrease in disease activity in the first trimester compared to preconception.

The previous studies demonstrated a flare after delivery, occurring between 3 and 12 months postpartum ${ }^{5,6}$. A reason they did not find the early flare demonstrated in our study could be that the women were not examined as early as 6 weeks postpartum. In our study, the early flare may be underestimated, considering that the percentage of women using bDMARD increased from $2 \%$ to $25 \%$. After 6 weeks postpartum, the percentage of women using bDMARD almost doubled, probably contributing to the decrease in disease activity between 6 weeks and 1 year postpartum. Overall, we cannot tell much about the natural course of the disease in the year after delivery, because DMARD was restarted in this period.

A change in DAS28 of $\geq 1.2$ is often regarded as clinically significant ${ }^{13}$. In our study, the difference in DAS28 between first trimester and 6 weeks postpartum, though statistically significant, was only 0.27 . With the percentage of women in remission decreasing from $72 \%$ to $53 \%$ in the same period, and the percentage with high disease activity doubling, we argue that this finding is clinically relevant despite the small change in estimated mean DAS28. This argument is supported by the fact that $22 \%$ of the women with data from both the third trimester and 6 weeks postpartum experienced clinically significant worsening according to the above definition, as opposed to only $4 \%$ experiencing clinically significant amelioration.

We found that women using oral steroids had significantly higher disease activity. These women were most probably prescribed steroids because of their high disease activity. Women using SSZ also tended to have higher disease activity. One reason might be that women with more serious diseases were continuing DMARD in pregnancy. We did not include basic characteristics such as maternal age, disease duration, and parity. These factors were constant throughout the period and thus should not influence disease activity during followup.

As expected, we found that functionality was worst in late pregnancy. Previous studies using HAQ have demonstrated the same findings in healthy pregnant women and pregnant women with $\mathrm{RA}^{9,15}$. As noted in these studies, it is not possible to distinguish the effect on functionality of pregnancy itself from the effect of the disease.

SF-36 has been used in a previous study of pregnant women with RA and ankylosing spondylitis (AS), demonstrating that women with RA experienced less pain during pregnancy while women with AS, like our study population, had unchanged bodily pain scores ${ }^{16}$. Further, both women with RA and women with AS, like our study population, reported stable mental health. Of note, in our population we found a significant improvement in reported mental health 6 weeks after delivery, at the same time when disease activity was highest. This is reassuring knowledge for women with JIA considering motherhood.

The most important strengths of our study are the prospective design, the use of a validated disease activity score, and the statistical method. The choice of DAS28-CRP3 as disease activity score was based on the widespread use of DAS28 in chronic arthritis in adults and the validation of DAS28-CRP3 in pregnant women with $\mathrm{RA}^{9}$. In pediatrics, there is increasing use of the Juvenile Arthritis Disease Activity Score ${ }^{1}$, but it has not been validated in adults or in pregnancy.

The mixed model approach made it possible to study how disease activity developed throughout a time period in the same subjects. The choice of method was suited to our relatively large study population, because it allows for missing data as long as they are missing at random. Choosing a model in which visits were nested within pregnancies and pregnancies were nested within women, we took into account that measurements within the same woman were correlated. Thus, we could include women with $>1$ pregnancy. We included 5 women who contributed DAS28 scores only postpregnancy, after confirming that results of analyses were substantially the same when excluding them (results not shown). One woman pregnant with twins was included, also after confirming that results were substantially the same when excluding the twin pregnancy (results not shown). MHAQ values originally had a skewed distribution, but we obtained normality through log transformation. When analyzing both original data and log transformed data in a linear mixed model, results were substantially the same (data not shown),

$$
\text { Personal non-commercial use only. The Journal of Rheumatology Copyright } \subset \text { C 2018. All rights reserved. }
$$


and for simplicity, we presented results from the original MHAQ data. Normality of residuals was checked by inspection of quantile-quantile plots.

Another strength of our study is that all women were diagnosed and treated by a rheumatologist in the Norwegian public healthcare system, which ensured proper diagnosis and equal health services.

Our study population was socioeconomically homogeneous - the majority of the women were white, in relationships, and had stable incomes.

The main limitation of our study was that not all women had data from all timepoints. Only $35 \%$ of the women were included before conception. It is possible that women with less severe diseases were less likely to be in contact with a rheumatologist before pregnancy. Consequently, missing values at the preconception visit were not missing entirely at random, and disease activity at this timepoint might be overestimated. Also, there could be a tendency toward milder disease in women missing the early visits of pregnancy, contributing to an overestimation of disease activity in pregnancy. However, this would strengthen our finding that there was a significant increase in disease activity between pregnancy and 6 weeks postpartum. When the analysis was performed, $17 \%$ of the women had not yet completed the postpartum followup. These registrations were missing at random.

Selection bias may influence our results in several ways. Even though inclusion in RevNatus is the norm in Norwegian rheumatology units, some women in longterm remission may be under the care of their general practitioner and therefore may not be included in the register. This could result in a selection of women with higher disease activity in our study. On the other hand, our study might not have included the women with the poorest health, because high disease activity, organ involvement, or comorbidities may exclude motherhood.

Our results cannot be directly generalized to countries with other healthcare systems. In countries with less focus on planning pregnancies in women with rheumatic diseases, disease activity would probably have been higher.

By narrowing the definition of visits (for example, defining first trimester as between 8 and 12 weeks of pregnancy), data would be more precise. The multicenter design and the practicalities of planning consultations made this difficult. The preconception visit was the visit least clearly defined and could theoretically be between 1 year and 1 week before conception. We chose 6 weeks postpartum as a reference point, because this was a well-defined nonpregnant timepoint with few missing DAS28 scores.

Another limitation of our study is that we did not have information about the women's JIA subtype. JIA subtypes have different pathophysiologies ${ }^{17}$, and may represent different patterns of disease activity during pregnancy. As shown in Table $1,<3 \%$ in our population had spondylo- arthritis (psoriatic arthritis and enthesitis-related arthritis combined), and only 1 woman had organ involvement, while $13 \%$ were RF-positive. According to a Norwegian study on disease progression of JIA into adulthood ${ }^{3}$, our study overestimated the proportion of patients positive for RF (3\% in the study) and underestimated the proportion with spondyloarthritis and systemic JIA.

In our study, which prospectively explored disease activity during and after pregnancy in women with JIA using a validated disease activity score, we found that the majority experienced low and relatively stable disease activity. A small but significant increase in disease activity 6 weeks postpartum calls for tight followup of women with JIA in the first weeks after delivery. Future research on pregnancy in subgroups of JIA would be of interest.

\section{ACKNOWLEDGMENT}

The authors thank Hege Svean Koksvik and Bente Jakobsen at the National Advisory Unit on Pregnancy and Rheumatic Diseases, Trondheim University Hospital, for the day-to-day management of RevNatus and practical help with collecting data from the register. We also thank Dr. Anne N. Bendvold, in private practice in Kristiansand, and the participating departments of rheumatology at the following hospitals for including patients in RevNatus: Betanien Hospital, Skien; Diakonhjemmet Hospital, Oslo; Haugesund Sanitetsforenings Rheumatism Hospital, Haugesund; Haukeland University Hospital, Bergen; Helse Førde, Førde Hospital, Førde; Helse Møre og Romsdal, Ålesund Hospital, Ålesund; Lillehammer Hospital for Rheumatic Diseases, Lillehammer; Nordland Hospital, Bodø; Oslo University Hospital Rikshospitalet, Oslo; Trondheim University Hospital, Trondheim; Sørlandet Hospital Kristiansand, Kristiansand; University Hospital of North Norway, Troms $\varnothing$; Vestre Viken Hospital, Drammen; Østfold Hospital, Moss.

\section{REFERENCES}

1. Nordal EB, Zak M, Aalto K, Berntson L, Fasth A, Herlin T, et al. Validity and predictive ability of the juvenile arthritis disease activity score based on CRP versus ESR in a Nordic population-based setting. Ann Rheum Dis 2012;71:1122-7.

2. Minden K. Adult outcomes of patients with juvenile idiopathic arthritis. Horm Res 2009;1:20-5.

3. Selvaag AM, Aulie HA, Lilleby V, Flato B. Disease progression into adulthood and predictors of long-term active disease in juvenile idiopathic arthritis. Ann Rheum Dis 2016;75:190-5.

4. de Man YA, Dolhain RJ, van de Geijn FE, Willemsen SP, Hazes JM. Disease activity of rheumatoid arthritis during pregnancy: results from a nationwide prospective study. Arthritis Rheum 2008;59:1241-8.

5. Ostensen M. The effect of pregnancy on ankylosing spondylitis, psoriatic arthritis, and juvenile rheumatoid arthritis. Am J Reprod Immunol 1992;28:235-7.

6. Musiej-Nowakowska E, Ploski R. Pregnancy and early onset pauciarticular juvenile chronic arthritis. Ann Rheum Dis 1999;58:475-80.

7. Petty RE, Southwood TR, Manners P, Baum J, Glass DN, Goldenberg J, et al. International League of Associations for Rheumatology classification of juvenile idiopathic arthritis: second revision, Edmonton, 2001. J Rheumatol 2004;31:390-2.

8. Prevoo ML, van 't Hof MA, Kuper HH, van Leeuwen MA, van de Putte LB, van Riel PL. Modified disease activity scores that include twenty-eight-joint counts. Development and validation in a prospective longitudinal study of patients with rheumatoid arthritis. Arthritis Rheum 1995;38:44-8. 
9. de Man YA, Hazes JM, van de Geijn FE, Krommenhoek C, Dolhain RJ. Measuring disease activity and functionality during pregnancy in patients with rheumatoid arthritis. Arthritis Rheum 2007; 57:716-22.

10. van Gestel AM, Prevoo ML, van 't Hof MA, van Rijswijk MH, van de Putte LB, van Riel PL. Development and validation of the European League Against Rheumatism response criteria for rheumatoid arthritis. Comparison with the preliminary American College of Rheumatology and the World Health

Organization/International League Against Rheumatism Criteria. Arthritis Rheum 1996;39:34-40.

11. Ware JE Jr. SF-36 health survey update. Spine 2000;25:3130-9.

12. Maska L, Anderson J, Michaud K. Measures of functional status and quality of life in rheumatoid arthritis: Health Assessment Questionnaire Disability Index (HAQ), Modified Health Assessment Questionnaire (MHAQ), Multidimensional Health Assessment Questionnaire (MDHAQ), Health Assessment Questionnaire II (HAQ-II), Improved Health Assessment Questionnaire (Improved HAQ), and Rheumatoid Arthritis Quality of Life (RAQoL). Arthritis Care Res 2011;63 Suppl 11:4-13.
13. van der Maas A, Lie E, Christensen R, Choy E, de Man YA, van Riel $\mathrm{P}$, et al. Construct and criterion validity of several proposed DAS28-based rheumatoid arthritis flare criteria: an OMERACT cohort validation study. Ann Rheum Dis 2013;72:1800-5.

14. Alpigiani MG, Salvati P, Vannati M, Siciliano C, Callegari S, Intra $\mathrm{C}$, et al. Pregnancy in women of childbearing age affected by juvenile idiopathic arthritis (JIA). Clin Exp Rheumatol 2011;29:416.

15. Barrett JH, Brennan P, Fiddler M, Silman AJ. Does rheumatoid arthritis remit during pregnancy and relapse postpartum? Results from a nationwide study in the United Kingdom performed prospectively from late pregnancy. Arthritis Rheum 1999; 42:1219-27.

16. Forger F, Ostensen M, Schumacher A, Villiger PM. Impact of pregnancy on health related quality of life evaluated prospectively in pregnant women with rheumatic diseases by the SF-36 health survey. Ann Rheum Dis 2005;64:1494-9.

17. Eisenstein EM, Berkun Y. Diagnosis and classification of juvenile idiopathic arthritis. J Autoimmun 2014;48-49:31-3. 